TITLE:

\title{
Energy dissipation in spiral vortex layers wrapped around a straight vortex tube
}

$\operatorname{AUTHOR}(S)$ :

Kawahara, G

\section{CITATION:}

Kawahara, G. Energy dissipation in spiral vortex layers wrapped around a straight vortex tube. PHYSICS OF FLUIDS 2005, 17(5): 055111.

\section{ISSUE DATE:}

2005-05

URL:

http://hdl.handle.net/2433/50071

\section{RIGHT:}

Copyright 2005 American Institute of Physics. This article may be downloaded for personal use only. Any other use requires prior permission of the author and the American Institute of Physics. 


\title{
Energy dissipation in spiral vortex layers wrapped around a straight vortex tube
}

\author{
Genta Kawahara ${ }^{\text {a) }}$ \\ Department of Aeronautics and Astronautics, and Advanced Research Institute of Fluid Science \\ and Engineering, Kyoto University, Kyoto 606-8501, Japan
}

(Received 5 October 2004; accepted 22 February 2005; published online 9 May 2005)

\begin{abstract}
Energy dissipation in spiral layers of high azimuthal vorticity around a straight vortex tube is investigated analytically. Asymptotic expressions of local and total viscous dissipation are obtained for the spiral vortex layers. When a vortex tube, which aligns with a uniform shear flow of a shear rate $S$, starts with a vortex filament of circulation $\Gamma$ at an initial instant $t=0$, it wraps and stretches background vorticity lines around itself to form double spiral vortex layers of intense dissipation. The contribution of the spiral layers to total dissipation per unit axial length is evaluated to be $1.29 \pi \nu^{2} S^{2}(\Gamma / 2 \pi \nu)^{4 / 3} t$ at large vortex Reynolds numbers $\Gamma / \nu \gg 1, \nu$ being the kinematic viscosity of fluid. There exists the critical time after which the contribution of the spirals to the total dissipation dominates that of the tube. If the tube is tilted at a small angle $\alpha$ in the direction of the uniform shear vorticity, the spirals around the tube are cross axially sheared into different shapes depending on the sign of $\alpha$, which leads to local reduction (or enhancement) of the energy dissipation in the spirals at $\alpha>0$ (or $<0$ ). The primary effect of the cross-axial shear on the total dissipation is shown to be $-\frac{1}{4} \alpha \nu S^{3} \Gamma \ln (\Gamma / 2 \pi \nu) t^{2}$ at $S t|\alpha| \ll 1$ for $\Gamma / \nu \gg 1$. The contribution to turbulent energy dissipation from spiral structures around a tubular vortex at a large-Reynolds-number limit is also discussed based upon recently reported direct numerical simulations and the present analysis. (C) 2005 American Institute of Physics. [DOI: 10.1063/1.1897011]
\end{abstract}

\section{INTRODUCTION}

Tube-like coherent structures of concentrated intense vorticity have commonly been observed in various classes of turbulent flows, e.g., isotropic turbulence, ${ }^{1}$ homogeneous shear turbulence, ${ }^{2}$ mixing layer turbulence, ${ }^{3}$ and near-wall turbulence. ${ }^{4}$ In homogeneous isotropic turbulence, among others, much effort has been devoted to characterization of tubular vortices. ${ }^{1,5-14}$ It has widely been accepted that tubelike elongated structures have a cross-sectional radius of the order of the Kolmogorov microscale, and individual tubes have length of the order ranging from the Taylor microscale to the integral scale of turbulence. They have lifetimes of the order of the large-eddy-turnover time. ${ }^{14}$ Since Tennekes ${ }^{15}$ proposal of a structural model of turbulence, in which all of turbulence kinetic energy was assumed to be dissipated by vortex tubes, the energy dissipation caused by tube-like structures in turbulence has been investigated numerically ${ }^{7,12}$ and analytically. ${ }^{16}$ Tubular vortices have been expected to be one of the typical dissipative structures in turbulence. In recent direct numerical simulations of isotropic turbulence, however, Jiménez and Wray ${ }^{14}$ have shown that tubular structures of strong axial vorticity, although responsible for most of the intermittency effects of the higher moments of velocity derivatives, are themselves responsible for only a negligible part of the energy dissipation. They found that strong tubular vortices, which have axial vorticity of $O\left(\omega_{\mathrm{rms}} R_{\lambda}^{1 / 2}\right)$, occupy a volume fraction of the order $R_{\lambda}^{-2}$, where $R_{\lambda}$ is the Taylor-microscale Reynolds number and $\omega_{\text {rms }}$ represents the

${ }^{a)}$ Electronic mail: gkawahara@ kuaero.kyoto-u.ac.jp rms vorticity of turbulence. This finding implies that the contribution rate to the total dissipation from strong tubes is of the order $R_{\lambda}^{-1}$, and therefore it should decrease rapidly with $R_{\lambda}$.

Another candidate for typical dissipative structures in turbulence is spiral layers of concentrated vorticity, ${ }^{17-19}$ which are traced back to Corrsin's ${ }^{20}$ layer-like model of turbulence. Although spiral structures in turbulence have been less understood, there are at least two possible mechanisms of their generation. If a vortex layer winds up into a vortex tube, ${ }^{21-23}$ the original layer forms double spirals around the tube. The vortex layers wound up in this way are the Lundgren spirals. ${ }^{17}$ If a vortex tube is in a weaker background vorticity field, on the other hand, the tube wraps and stretches vorticity lines around itself to form double spiral vortex layers. ${ }^{18,19}$ We note that in the former mechanism, i.e., the Lundgren spirals, the vorticity in spirals aligns with a tube, while in the latter the vorticity is dominated by the cross-axial component because of vorticity stretching and intensification. Therefore, these two classes of spirals are quite distinct from each other. Lundgren ${ }^{17}$ has obtained a largetime asymptotic solution to the Navier-Stokes equations for the spiral layers of the axial vorticity subjected to an axisymmetric irrotational strain and shown that his spiral vortices exhibit the energy spectrum with a range of a Kolmogorov $k^{-5 / 3}$ falloff (see also the works of Lundgren ${ }^{24}$ and Gilbert ${ }^{25}$ ). The spirals of the cross-axial vorticity in the axisymmetric straining flow have a $k^{-7 / 3}$ spectrum at large wavenumbers, ${ }^{26,27}$ and the energy dissipation for the spirals of the axial and the cross-axial vorticities can be of comparable order. ${ }^{26}$ Recently, in their direct numerical simulation 
of isotropic turbulence, Kida and Miura ${ }^{28}$ have found the existence of double spiral vortex layers with the dominant azimuthal compoment of vorticity, that is, the latter class of spirals. Horiuti $\mathrm{i}^{29,30}$ has proposed a classification method for layer and tube structures in turbulence and observed a vortex layer with the vorticity perpendicular to that of a tube.

In this paper, we investigate analytically energy dissipation in spiral vortex layers of intense azimuthal vorticity that are formed through wrap and stretch of vorticity lines around a vortex tube. We introduce a highly simplified model in order to obtain analytical solutions for the full description of viscous dissipation caused by spiral vortex layers. In this model, a straight diffusing vortex tube, which starts with a vortex filament at initial time, is embedded in a uniform shear flow, and the tube is tilted in the direction of the uniform shear vorticity at a small angle $\alpha$ from the streamwise direction. In isotropic turbulence as well as sheared turbulence, straightly elongated tubular vortices of intense vorticity commonly exist in a background vorticity field, and they generally undergo a cross-axial nonaxisymmetric strain. In turbulent shear flows, for instance, longitudinal tubular structures are often observed to slightly tilt in the spanwise direction from the streamwise direction, ${ }^{2,4}$ so that the tubes and spiral layers around them may be sheared cross axially. The flow to be discussed in this paper, therefore, could be regarded as one of the simplest models of essential flow structures around a tube-like vortex in turbulence.

Basic equations for the analytical model and a viscous dissipation rate are described in Sec. II. We analyze the energy dissipation for spiral vortex layers around a longitudinal tube for $\alpha=0$ to elucidate the contribution of the spirals to total dissipation in Sec. III. The role of spiral structures around tubular vortices in turbulent energy dissipation is also discussed based upon recently reported direct numerical simulations ${ }^{1,14,31}$ and the present analysis. In Sec. IV, the energy dissipation in the spirals around a slightly tilted vortex tube is analyzed asymptotically at early-time evolution $S t|\alpha| \ll 1$ to demonstrate the effect of cross-axial shear on the spirals and their total dissipation. Section V is devoted to the concluding remarks. Preliminary versions of parts of the present manuscript appeared previously as Refs. 32 and 33.

\section{FORMULATION}

Let us consider an incompressible viscous flow around an infinitely straight vortex tube. At an initial instant $t=0$, a straight vortex filament of circulation $\Gamma(>0)$ is set in a uniform shear flow. The velocity and the vorticity of the uniform shear flow are, respectively, $\boldsymbol{S} Y \boldsymbol{e}_{X}$ and $-\boldsymbol{S} \boldsymbol{e}_{Z}$, where $S(>0)$ is a constant shear rate, and $\boldsymbol{e}_{X}$ and $\boldsymbol{e}_{Z}$ denote unit vectors in the streamwise $(X)$ and the spanwise $(Z)$ directions, respectively. The origin $O$ of the coordinate system $O X Y Z$ (and also Oxyz below) is located on the vortex filament. The filament is inclined at an angle $\alpha$ from the $X$ axis on the plane $Y=0$, and at $t>0$ it will be diffused into a vortex tube of a finite radius under the action of viscosity. In the following, we shall formulate the problem by using another coordinate system $O x y z$. The coordinate system $O x y z$ is defined by rotating the original coordinate system $O X Y Z$ by the angle $\alpha$ around the $Y$ axis so that the $x$ axis can coincide with the central axis of the vortex tube. The vorticity of the tube is taken to be pointed in the positive $x$ direction. Hereafter, the $x$ direction is called the axial direction, while the $y$ and the $z$ directions are called the cross-axial directions. If the inclination angle $\alpha$ is positive (or negative), the tube has the spanwise vorticity component of the same (or opposite) sign as that of the uniform shear flow. Hereafter, the vortex tube of $\alpha>0$ (or $<0$ ) is referred to as cyclone (or anticyclone). The cyclone and the anticyclone undergo the crossaxial shear as well as the axial shear. At the neutral case of $\alpha=0$, which will first be considered in Sec. III, the vortex tube aligns with the uniform shear flow, so that the coordinate system $O x y z$ coincides with the original one $O X Y Z$. In this case the vortex tube is not subjected to the cross-axial shear.

\section{A. Basic equations}

Although the axial dependence of the flow around tubular structures would affect their dynamics, ${ }^{34}$ intense vortex tubes in many turbulent flows are likely elongated rather straight so that the essential process of their dynamics may be approximately described under the assumption of uniformity of the flow field along the vortex tube. If we suppose that the velocity and the pressure fields are uniform along the central axis of the tube, i.e., the $x$ axis, the velocity $\boldsymbol{v}(y, z, t)$ and the vorticity $\boldsymbol{\omega}(y, z, t)$ can be expressed using the streamfunction $\psi(y, z, t)$ and the axial velocity $u(y, z, t)$, respectively, as

$$
\begin{aligned}
& \boldsymbol{v}=u \boldsymbol{e}_{x}+\frac{\partial \psi}{\partial z} \boldsymbol{e}_{y}+\left(-\frac{\partial \psi}{\partial y}+S y \sin \alpha\right) \boldsymbol{e}_{z}, \\
& \boldsymbol{\omega}=\left(-\nabla^{2} \psi+S \sin \alpha\right) \boldsymbol{e}_{x}+\frac{\partial u}{\partial z} \boldsymbol{e}_{y}-\frac{\partial u}{\partial y} \boldsymbol{e}_{z},
\end{aligned}
$$

where $\boldsymbol{e}_{x}, \boldsymbol{e}_{y}$, and $\boldsymbol{e}_{z}$ denote unit vectors in the $x$, the $y$, and the $z$ directions, respectively, and $\nabla^{2}=\partial^{2} / \partial y^{2}+\partial^{2} / \partial z^{2}$ is a two-dimensional Laplacian operator. In (1) and (2), $S y \sin \alpha$ represents the cross-axial shear flow, which originates from the uniform shear flow, and $S \sin \alpha$ represents its vorticity. The streamfunction appears in the axial component of the vorticity to represent the vortex tube, while the axial velocity appears in the cross-axial components of the vorticity to represent the spiral vortex layers. Note that isocontours of the axial velocity $u$ on the cross-axial $[(y, z)-]$ plane are consistent with the projections of vorticity lines on that plane.

The streamfunction and the axial velocity obey the vorticity equation

$$
\left(\frac{\partial}{\partial t}-\nu \nabla^{2}\right) \nabla^{2} \psi-\frac{\partial\left(\psi, \nabla^{2} \psi\right)}{\partial(y, z)}=-S y \sin \alpha \frac{\partial}{\partial z} \nabla^{2} \psi
$$

and the Navier-Stokes equation

$$
\left(\frac{\partial}{\partial t}-\nu \nabla^{2}\right) u-\frac{\partial(\psi, u)}{\partial(y, z)}=-S y \sin \alpha \frac{\partial u}{\partial z},
$$




$$
\frac{\partial(\beta, \gamma)}{\partial(y, z)}=\frac{\partial \beta}{\partial y} \frac{\partial \gamma}{\partial z}-\frac{\partial \beta}{\partial z} \frac{\partial \gamma}{\partial y}
$$

is the Jacobian determinant and $\nu$ is the kinematic viscosity of fluid. The right-hand side of (3) and (4) comes from the advection by the cross-axial shear flow. Equations (3) and (4) are supplemented by the initial conditions as

$$
\psi(y, z, t=0)=-\frac{\Gamma}{2 \pi} \ln r, \quad u(y, z, t=0)=S y \cos \alpha,
$$

where $r=\left(y^{2}+z^{2}\right)^{1 / 2}$ denotes a radial distance from the $x$ axis. The boundary conditions to be imposed at $r \rightarrow \infty$ are that

$$
\frac{\partial \psi}{\partial z}=-\frac{\partial \psi}{\partial y}=0, \quad u=S y \cos \alpha .
$$

In addition to the boundary conditions (7) at infinity, $\psi$ and $u$ are assumed to be regular at $r=0$.

By introducing the plane polar coordinates $(r, \theta)$ with $y=r \cos \theta$ and $z=r \sin \theta$, (3) and (4) are rewritten as

$$
\begin{aligned}
\left(\frac{\partial}{\partial t}\right. & \left.-\nu \nabla^{2}\right) \nabla^{2} \psi-\frac{1}{r} \frac{\partial\left(\psi, \nabla^{2} \psi\right)}{\partial(r, \theta)} \\
& =-\frac{1}{2} S \sin \alpha\left(r \sin 2 \theta \frac{\partial}{\partial r}+\cos 2 \theta \frac{\partial}{\partial \theta}+\frac{\partial}{\partial \theta}\right) \nabla^{2} \psi, \\
\left(\frac{\partial}{\partial t}\right. & \left.-\nu \nabla^{2}\right) u-\frac{1}{r} \frac{\partial(\psi, u)}{\partial(r, \theta)} \\
& =-\frac{1}{2} S \sin \alpha\left(r \sin 2 \theta \frac{\partial}{\partial r}+\cos 2 \theta \frac{\partial}{\partial \theta}+\frac{\partial}{\partial \theta}\right) u,
\end{aligned}
$$

where $\nabla^{2}=\partial^{2} / \partial r^{2}+r^{-1} \partial / \partial \theta+\partial^{2} / \partial \theta^{2}$. These two equations, (8) and (9), are our basic equations for the analyses in Secs. III and IV.

\section{B. Viscous dissipation rate}

We next consider an energy dissipation rate. Let $s_{i j}(i, j$ $=1,2,3)$ denote a rate-of-strain tensor in the coordinate system $O x y z$, the elements of which are

$$
\left\{s_{i j}\right\}=\left(\begin{array}{ccc}
0 & \frac{1}{2} \frac{\partial u}{\partial y} & \frac{1}{2} \frac{\partial u}{\partial z} \\
\frac{1}{2} \frac{\partial u}{\partial y} & \frac{\partial^{2} \psi}{\partial z \partial y} & -\frac{1}{2}\left(\frac{\partial^{2}}{\partial y^{2}}-\frac{\partial^{2}}{\partial z^{2}}\right) \psi+\frac{1}{2} S \sin \alpha \\
\frac{1}{2} \frac{\partial u}{\partial z} & -\frac{1}{2}\left(\frac{\partial^{2}}{\partial y^{2}}-\frac{\partial^{2}}{\partial z^{2}}\right) \psi+\frac{1}{2} S \sin \alpha & -\frac{\partial^{2} \psi}{\partial y \partial z}
\end{array}\right)
$$

The viscous dissipation rate per unit mass and unit time is then given by

$$
\Phi=2 \nu s_{i j} s_{i j}=\nu S^{2}+D_{T}+D_{S},
$$

where the first term $\nu S^{2}$ comes from the background uniform shear flow, while the second

$$
\begin{aligned}
D_{T}(r, \theta, t)= & \nu\left(\frac{\partial^{2} \psi}{\partial r^{2}}-\frac{1}{r} \frac{\partial \psi}{\partial r}-\frac{1}{r^{2}} \frac{\partial^{2} \psi}{\partial \theta^{2}}\right)^{2} \\
& +\nu \frac{4}{r^{2}}\left(\frac{\partial^{2} \psi}{\partial r \partial \theta}-\frac{1}{r} \frac{\partial \psi}{\partial \theta}\right)^{2} \\
& -2 \nu S \sin \alpha\left(\frac{\partial^{2} \psi}{\partial r^{2}}-\frac{1}{r} \frac{\partial \psi}{\partial r}-\frac{1}{r^{2}} \frac{\partial^{2} \psi}{\partial \theta^{2}}\right) \cos 2 \theta \\
& +4 \nu S \sin \alpha \frac{1}{r}\left(\frac{\partial^{2} \psi}{\partial r \partial \theta}-\frac{1}{r} \frac{\partial \psi}{\partial \theta}\right) \sin 2 \theta
\end{aligned}
$$

and the third

$$
D_{S}(r, \theta, t)=\nu\left[\left(\frac{\partial u}{\partial r}\right)^{2}+\frac{1}{r^{2}}\left(\frac{\partial u}{\partial \theta}\right)^{2}-S^{2} \cos ^{2} \alpha\right]
$$

correspond to the contributions from the vortex tube and spiral vortex layers, respectively. In (12), the first and second terms represent the contribution of the tube itself, while the third and the fourth represent a consequence of the interaction between the tube and the background cross-axial shear flow. Note that in (13), $(\partial u / \partial r)^{2}+r^{-2}(\partial u / \partial \theta)^{2}$ represents the square of the cross-axial vorticity, and the contribution of the background axial shear flow has been subtracted.

\section{DISSIPATION FOR $\alpha=0$ : TUBE VERSUS SPIRALS}

Let us consider the energy dissipation around the vortex tube aligned with the uniform shear flow at $\alpha=0$. The largeReynolds-number asymptotic forms of the velocity and the vorticity fields for the neutral case of $\alpha=0$ have already been obtained by Moore ${ }^{18}$ and Kawahara, Kida, Tanaka, and Yanase. ${ }^{19}$ We first describe Moore's asymptotic solution briefly.

For $\alpha=0$, the solution to the vorticity equation (8) under the initial and boundary conditions (6) and (7) is uniquely determined as

$$
\psi(r, t)=-\frac{\Gamma}{2 \pi} \int_{0}^{\eta} \frac{1-e^{-\xi^{2}}}{\xi} d \xi
$$

except for an additive constant, which does not affect the flow, where 


$$
\eta=\frac{1}{2} r(\nu t)^{-1 / 2}
$$

is a similarity variable. The corresponding axial vorticity is given by

$$
-\nabla^{2} \psi(r, t)=\frac{\Gamma}{4 \pi \nu t} e^{-\eta^{2}}
$$

The streamfunction (14) and the axial vorticity (16) represent an axisymmetric diffusing vortex tube.

We next consider the axial velocity $u$. Let us seek the solution to the Navier-Stokes equation (9), written in a separation-of-variable form in the similarity variable $\eta$ and the angular coordinate $\theta$, as

$$
u(r, \theta, t)=\operatorname{Sr} \operatorname{Re}\left[f(\eta) e^{-i \theta}\right]
$$

Substitution of (14) and (17) into (9) yields

$$
f^{\prime \prime}+\left(2 \eta+\frac{3}{\eta}\right) f^{\prime}+i \frac{\Gamma}{2 \pi \nu} \frac{1-e^{-\eta^{2}}}{\eta^{2}} f=0
$$

where hereafter the prime is used to denote differentiation with respect to $\eta$. Boundary conditions to be imposed are that $r f(\eta)$ is regular at $\eta=0$ and that $f(\infty)=1$ [see (6) and (7)]. At $\Gamma / \nu \gg 1$, the asymptotic solution to (18) has been obtained in the far region $\eta \gg(\Gamma / \nu)^{1 / 4}$ from the vortex tube by Moore $^{18}$ and Kawahara, Kida, Tanaka, and Yanase ${ }^{19}$ as

$$
f \approx \exp \left(i \frac{R_{\Gamma}}{4 \eta^{2}}-\frac{R_{\Gamma}^{2}}{48 \eta^{6}}\right)
$$

where

$$
R_{\Gamma}=\frac{\Gamma}{2 \pi \nu}
$$

denotes the vortex Reynolds number. The solution $f$ is exponentially small at $\Gamma / \nu \gg 1$ up to the region $\eta \sim(\Gamma / \nu)^{1 / 4}$. Note that the asymptotic expression (19) is for the far region and thus it is independent of the inner structure of the vortex tube.

\section{A. Structures of dissipation rate}

We now investigate the viscous dissipation around the vortex tube. By substituting the solution (14) into the dissipation rate (12) for $\alpha=0$, we obtain the dissipation for the tube,

$$
D_{T}=\nu\left(\frac{\Gamma}{4 \pi \nu t}\right)^{2}\left[\frac{1}{\eta^{2}}-\left(1+\frac{1}{\eta^{2}}\right) e^{-\eta^{2}}\right]^{2} .
$$

For the dissipation caused by the spiral vortex layers, on the other hand, the square of the cross-axial vorticity is expressed in terms of the function $f$, defined by (17), as

$$
\begin{aligned}
\left(\frac{\partial u}{\partial r}\right)^{2}+\frac{1}{r^{2}}\left(\frac{\partial u}{\partial \theta}\right)^{2}= & S^{2}\left[\frac{1}{2}\left|\eta f^{\prime}\right|^{2}+\frac{1}{2} \eta\left(|f|^{2}\right)^{\prime}+|f|^{2}\right] \\
& +S^{2} \operatorname{Re}\left[\eta f^{\prime}\left(f+\frac{1}{2} \eta f^{\prime}\right) e^{-2 i \theta}\right],
\end{aligned}
$$

and thus we substitute (22) into the dissipation rate (13) at $\alpha=0$ to obtain

$$
\begin{aligned}
D_{S}= & \nu S^{2}\left[\frac{1}{2}\left|\eta f^{\prime}\right|^{2}+\frac{1}{2} \eta\left(|f|^{2}\right)^{\prime}+|f|^{2}-1\right] \\
& +\nu S^{2} \operatorname{Re}\left[\eta f^{\prime}\left(f+\frac{1}{2} \eta f^{\prime}\right) e^{-2 i \theta}\right] .
\end{aligned}
$$

Figures 1(a) and 1(b) show the spatial distributions, in the cross-axial plane, of the magnitude of the cross-axial vorticity $\left[(\partial u / \partial r)^{2}+r^{-2}(\partial u / \partial \theta)^{2}\right]^{1 / 2}$ and of the viscous dissipation $D_{T}+D_{S}$ at $\alpha=0$ for moderate Reynolds number $\Gamma /(2 \pi \nu)=100$. In their direct numerical simulations of isotropic turbulence, Jiménez, Wray, Saffman, and Ragallo ${ }^{1}$ reported that the vortex Reynolds number of intense tubular vortices is increased as $\Gamma /(2 \pi \nu) \sim R_{\lambda}^{1 / 2}$, and its average is 20-34 at $R_{\lambda}=36-168$. Kida and Makihara ${ }^{31}$ recently presented the statistics of low-pressure vortices which are identified without any threshold ${ }^{35}$ in isotropic turbulence, and the average of $\Gamma /(2 \pi \nu)$ for their vortices are 17-20 at $R_{\lambda}$ $=82-174$. The cross-axial vorticity (a) and the viscous dissipation (b) have been obtained at time $S t=2.94$ by numerically solving the full equation (18). This is the critical time at which the contribution of the spirals [i.e., $D_{S}$ in (23)] to the total dissipation coincides with that of the tube [i.e., $D_{T}$ in (21)], as will be discussed in Sec. III B. Figures 1(c), 1(d), 1(e), and 1(f) are, respectively, for the cyclone and the anticyclone which will be discussed in Sec. IV. In Figs. 1(a), 1(c), and 1(e), a level of the vorticity magnitude is represented by color: the red is the highest $(4 S)$ and the blue is the lowest (i.e., null). In Figs. 1(b), 1(d), and 1(f) a level of the dissipation is also represented by color: the red is the highest $\left(27 \nu S^{2}\right)$ and the blue is the lowest (i.e., null). The isocontours of the axial velocity are also shown by the solid curves. The diagonal length of each panel is $40(\nu t)^{1 / 2}$, or equivalently 20 in the similarity variable $\eta$.

It can be seen in Fig. 1(a) that the vortex tube, which is located at the center of the panel, wraps and stretches the background vorticity lines (isocontours of $u$ ) around itself to form double spiral layers of high cross-axial vorticity. The vorticity lines run along the spirals so that the azimuthal component can be dominant in the cross-axial vorticity. The double spirals have the azimuthal vorticity of opposite sign to each other. The spirals in Fig. 1(a) are remarkably similar to those observed in real turbulence [cf. Fig. 1(c) in the paper of Kida and Miura $\left.{ }^{28}\right]$. We can see that the cross-axial vorticity disappears from the central region near the vortex tube. In the near region, the spirals of the opposite-signed azimuthal vorticities are wrapped so tightly that cross diffusion, i.e., viscous annihilation, is enhanced to smooth out the spirals. $^{18,19}$ 
(a)

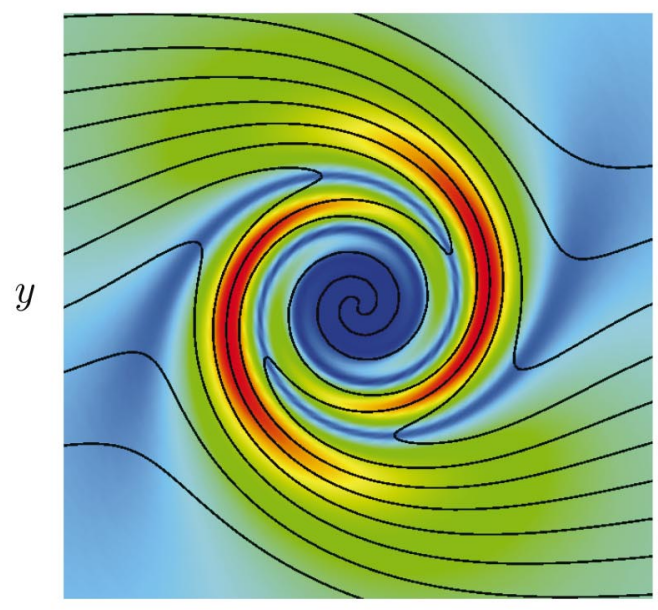

$z$

(c)

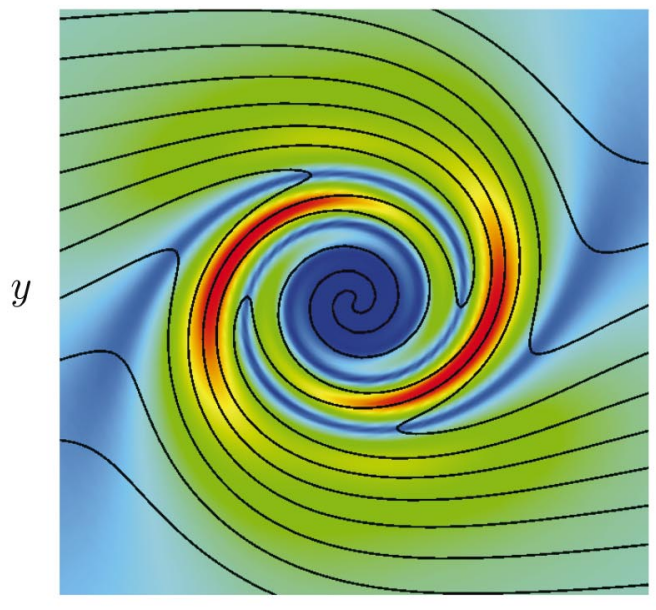

$z$

(e)

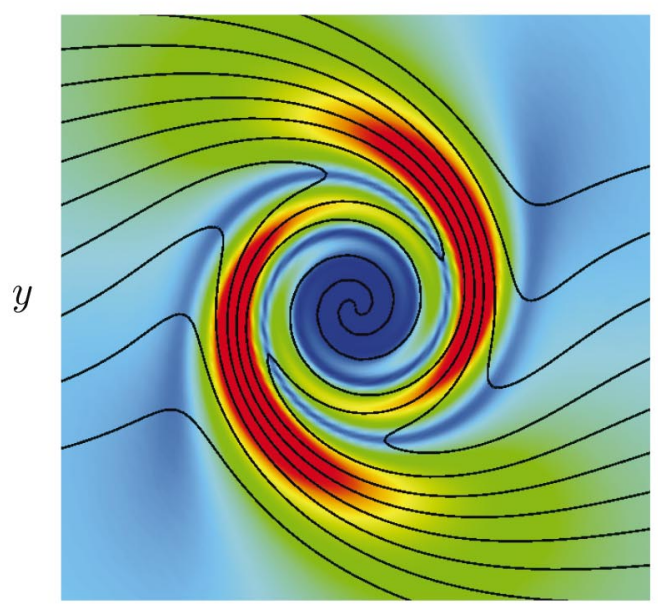

$z$ (b)

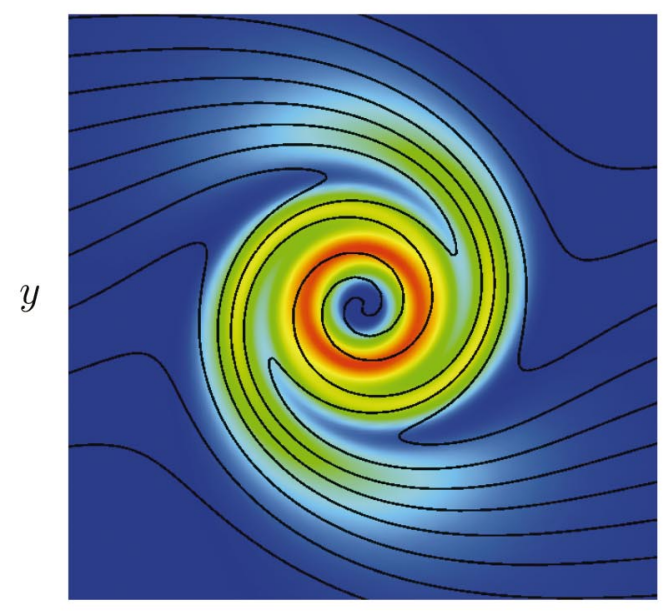

$z$

(d)

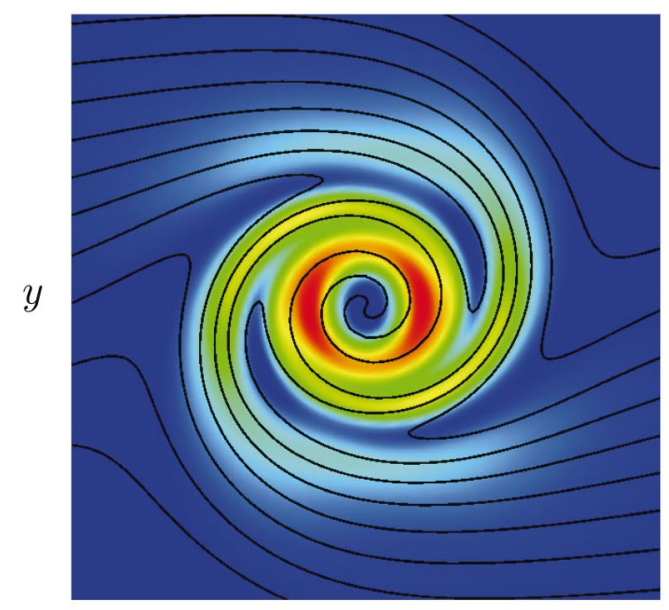

z

(f)

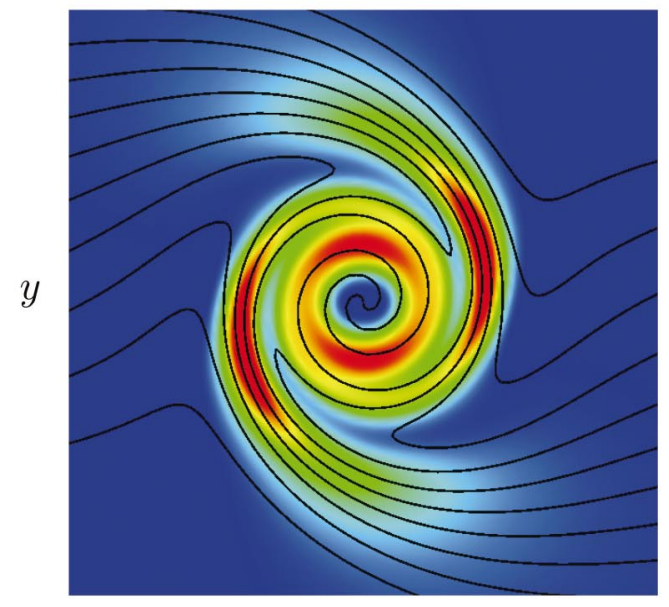

z

FIG. 1. (Color). Spatial distributions, in the cross-axial plane, of the magnitude of the cross-axial vorticity and of the viscous dissipation rate at the critical time $S t=2.94$ for vortex Reynolds number $\Gamma /(2 \pi \nu)=100$. [(a) and (b)] The neutral case $\alpha=0$. [(c) and (d)] The cyclonic case $\alpha=+0.2$. [(e) and (f)] The anticyclonic case $\alpha=-0.2$. In (a), (c), and (e), the magnitude of the cross-axial vorticity $\left[(\partial u / \partial r)^{2}+r^{-2}(\partial u / \partial \theta)^{2}\right]^{1 / 2}$ is shown by color: red is the highest $(4 S)$ and blue is the lowest (i.e., null). In (b), (d), and (f), the viscous dissipation rate $D_{T}+D_{S}$ is shown by color: red is the highest $\left(27 \nu S^{2}\right)$ and blue is the lowest (i.e., null). The solid curves represent the isocontours of the axial velocity $u$. Contour levels are $u=n(\nu S)^{1 / 2}[n=-20(4) 20]$. The diagonal length of each panel is $40(\nu t)^{1 / 2}$. 
In the corresponding viscous dissipation rate [Fig. 1(b)], there are two spatial structures of intense dissipation. One is the circular ring near the center, which is generated through a swirling flow induced by a vortex tube, i.e., $D_{T}$. The other is the two spirals around the ring, which represent intense dissipation caused by an axial flow induced by wrapped spiral layers of high azimuthal vorticity, i.e., $D_{S}$. Although the local energy dissipation in the spirals is lower than that in the ring, the volume per unit axial length of the spirals is larger than that of the ring so that the contributions to total-energy dissipation from the spirals and the tube can be consistent at the critical time, as will be shown below.

\section{B. Total dissipation and critical time}

Kawahara, Kida, Tanaka, and Yanase ${ }^{19}$ intuitively argued the possibility that the total dissipation for spiral vortex layers would be comparable to that for a vortex tube even at large vortex Reynolds numbers $\Gamma / \nu \gg 1$. Here, we strictly examine the total dissipation per unit axial length for the spirals in comparison with that for the tube.

The contribution of the tube to the total dissipation is evaluated, from (21), as

$$
\begin{aligned}
\int_{0}^{\infty} \int_{0}^{2 \pi} D_{T} r d r d \theta & =\frac{\Gamma^{2}}{2 \pi t} \int_{0}^{\infty}\left[\frac{1}{\eta^{2}}-\left(1+\frac{1}{\eta^{2}}\right) e^{-\eta^{2}}\right]^{2} \eta d \eta \\
& =\frac{\Gamma^{2}}{8 \pi t}
\end{aligned}
$$

which decreases in time $t$ because the tube is diffused as time progresses. The contribution of the spirals is, on the other hand, expressed, using (23), as

$$
\int_{0}^{\infty} \int_{0}^{2 \pi} D_{S} r d r d \theta=8 \pi \nu^{2} S^{2} I_{0} t
$$

where

$$
I_{0}=\int_{0}^{\infty}\left[\frac{1}{2}\left|\eta f^{\prime}\right|^{2}+\frac{1}{2} \eta\left(|f|^{2}\right)^{\prime}+|f|^{2}-1\right] \eta d \eta
$$

The contribution, (25), of the spirals increases linearly in time $t$ in contrast with (24) for the tube (see below for physical interpretation). At $\Gamma / \nu \gg 1$, by using the asymptotic solution (19) the primary order of the integral (26) can be estimated as

$$
\begin{aligned}
I_{0} & \approx 2^{-3}\left(\frac{\Gamma}{2 \pi \nu}\right)^{4 / 3} \int_{0}^{\infty} \frac{1}{\xi^{3}} \exp \left(-\frac{1}{24 \xi^{6}}\right) d \xi \\
& =2^{-3} 3^{-2 / 3} \Gamma\left(\frac{1}{3}\right)\left(\frac{\Gamma}{2 \pi \nu}\right)^{4 / 3},
\end{aligned}
$$

where $\Gamma(\cdot)$ is the gamma function. For $\Gamma / \nu \gg 1$, therefore, we can evaluate the primary contribution of the spirals as

$$
\int_{0}^{\infty} \int_{0}^{2 \pi} D_{S} r d r d \theta \approx 1.29 \pi \nu^{2} S^{2}\left(\frac{\Gamma}{2 \pi \nu}\right)^{4 / 3} t .
$$

Because the asymptotic expression (28) has been obtained from the solution (19) for the far region, the expression (28) does not depend on the inner structure of a vortex tube. Fig-

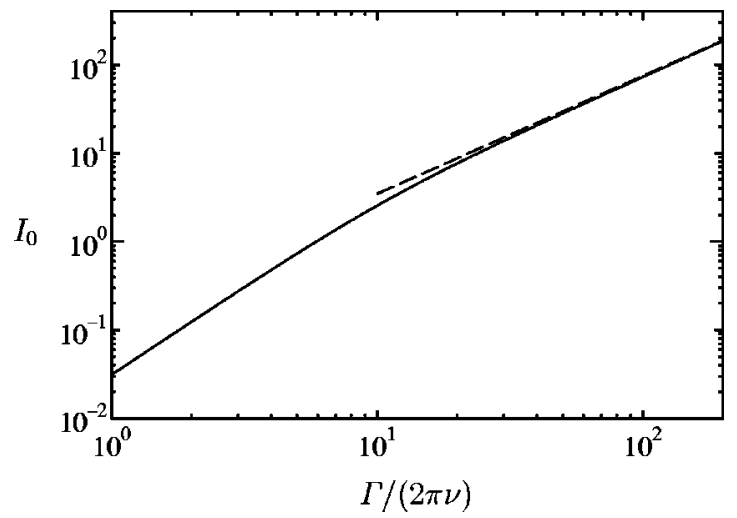

FIG. 2. Integral $I_{0}$ in (26) against vortex Reynolds number $\Gamma /(2 \pi \nu)$. The solid curve represents the numerical computation of (26) for the numerical solution $f$ to (18). The dashed line denotes the large-Reynolds-number asymptotics (27).

ure 2 compares the large-Reynolds-number asymptotic form (27) with the numerical computation of (26) for the numerical solution $f$ to the full equation (18). We can confirm that at large $\Gamma / \nu$, say $\Gamma /(2 \pi \nu) \geq 20-30$, the integral (26) is described satisfactorily by the asymptotic form (27), implying that the contribution to the total dissipation is dominated by the spirals in the far region. The value of the vortex Reynolds numbers for tubular structures observed in turbulence is around 20-30 at Taylor-microscale Reynolds numbers $R_{\lambda}$ $\approx 100$. ${ }^{1,31}$ A minimum Reynolds number of $R_{\lambda} \approx 100$ is required by fully developed turbulent states, ${ }^{36}$ in which the spiral structures could be represented by the asymptotic solution and the spirals in the far region might have dominant contribution to the dissipation. The total-energy dissipation for the spirals is increased proportionally to $(\Gamma / 2 \pi \nu)^{4 / 3}$ with increasing $\Gamma /(2 \pi \nu)$, as expressed in (28). This order of the contribution to the total dissipation from the spiral structures will be interpreted physically below.

As demonstrated above, the total dissipation for the vortex tube decreases with $t$, while that for the spiral vortex layers increases. Accordingly there exists the critical time at which the total dissipation for the spirals catches up with that for the tube. Let us evaluate the critical time $t_{c}$. By equating (24) with (25), we obtain the critical time

$$
S t_{c}=\frac{1}{4} I_{0}^{-1 / 2} \frac{\Gamma}{2 \pi \nu} .
$$

At large $\Gamma / \nu$, substitution of the primary order, (27), of the integral $I_{0}$ into (29) yields

$$
S t_{c}=0.623\left(\frac{\Gamma}{2 \pi \nu}\right)^{1 / 3} \text {. }
$$

The Reynolds-number dependence of the critical time $t_{c}$ is shown in Fig. 3. At time $t>t_{c}$ the contribution of the spiral vortex layers to the total dissipation dominates that of the vortex tube. Although the critical time increases gradually as the vortex Reynolds number is increased, it stays at a relatively low level $S t_{c}=O(1)$ [e.g., $S t_{c}=2.94$ even for $\Gamma /(2 \pi \nu)=100]$. It follows from the asymptotic form (30) that at large Reynolds numbers the critical time increases 


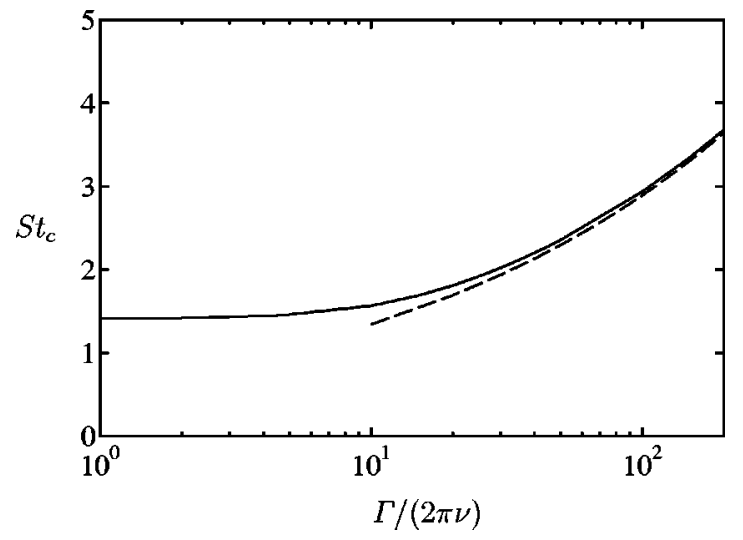

FIG. 3. Critical time $t_{c}$ in (29) against vortex Reynolds number $\Gamma /(2 \pi \nu)$. At time $t>t_{c}$ the contribution of spiral vortex layers to total dissipation exceeds that of a vortex tube. The solid curve represents the numerical result for (29). The dashed curve denotes the large-Reynolds-number asymptotics (30).

quite slowly as $(\Gamma / 2 \pi \nu)^{1 / 3}$. The above results lead us to the conclusion that a dominant contributor to the total-energy dissipation is the spiral vortex layers around the diffusing vortex tube rather than the tube itself at the later stage of time evolution.

Now we discuss the physical mechanism that determines the order of the energy dissipation caused by spiral structures around a vortex tube. Let us first recall Kawahara, Kida, Tanaka, and Yanase's ${ }^{19}$ intuitive argument about the structures of the velocity and the vorticity for the spiral vortex layers. In the far region from a vortex tube, we can regard the tube as a vortex filament. If we consider an inviscid case, the form of the spirals should be determined by the angular velocity, $\Gamma /\left(2 \pi r^{2}\right)$, of the swirling flow induced by the filament [see (6)] as $\theta-\Gamma t /\left(2 \pi r^{2}\right)=$ const. The radial spacing $\Delta r$ of the spirals is given by the change of $r$ per the angular change $\Delta \theta \approx 2 \pi$, so that we have $\left(\Gamma t / r^{3}\right) \Delta r \sim \Delta \theta \sim 1$, or equivalently $\Delta r \sim r^{3} /(\Gamma t)$. The azimuthal vorticity (or the radial gradient of the axial velocity) is then estimated as $u / \Delta r$ $\sim S r / \Delta r \sim S \Gamma t / r^{2}$. It turns out that in the inviscid spirals, their spacing would be infinitely small and thus their vorticity would be infinitely large at smaller $r$ because of the infinite wrap and stretch of the vorticity lines near the filament. In reality, however, the viscosity plays a role in the region where the spacing $\Delta r$ is comparable with the viscous length scale $(\nu t)^{1 / 2}$, that is, $r^{3} /(\Gamma t) \sim(\nu t)^{1 / 2}$ or equivalently $r$ $\sim(\Gamma / \nu)^{1 / 3}(\nu t)^{1 / 2}$. In this region the enhancement of the azimuthal vorticity is saturated by the effect of the viscosity, and consequently the azimuthal vorticity attains a maximum $u / \Delta r \sim S(\Gamma / \nu)^{1 / 3}$ whereas the cross-axial vorticity is expelled from the inner region $r \ll(\Gamma / \nu)^{1 / 3}(\nu t)^{1 / 2}$.

At $r \sim(\Gamma / \nu)^{1 / 3}(\nu t)^{1 / 2}$ the energy dissipation rate for the spirals also attains a maximum $\nu(u / \Delta r)^{2} \sim \nu S^{2}(\Gamma / \nu)^{2 / 3}$. The volume per unit axial length of the spirals with highest dissipation, i.e., the strongest spirals in the region $r$ $\sim(\Gamma / \nu)^{1 / 3}(\nu t)^{1 / 2}$, is of the order $(\Gamma / \nu)^{2 / 3} \nu t$, so that the primary contribution to total dissipation from the spirals is estimated as

$$
\nu\left(\frac{u}{\Delta r}\right)^{2}\left(\frac{\Gamma}{\nu}\right)^{2 / 3} \nu t \sim \nu^{2} S^{2}\left(\frac{\Gamma}{\nu}\right)^{4 / 3} t
$$

which is consistent with the order of the exact expression (28).

Let us next discuss the energy dissipation of a tubular structure. In the case of a diffusing vortex tube, its radius is of the order $(\nu t)^{1 / 2}$ and thus an azimuthal shear rate (or axial vorticity) is of the order $\Gamma /(\nu t)$, so that the dissipation rate is of the order $\nu(\Gamma / \nu t)^{2}$ and therefore the total dissipation is estimated as $\nu(\Gamma / \nu t)^{2} \nu t \sim \Gamma^{2} / t$. When the total dissipation for the diffusing tube is comparable to that for the spirals, $\nu^{2} S^{2}(\Gamma / \nu)^{4 / 3} t$, we have the critical time $S t_{c} \sim(\Gamma / \nu)^{1 / 3}$, which is consistent with the exact estimate (30). If we suppose the diffusing vortex tube subject to the axial stretching of a strain rate $\sigma$, it approaches the Burgers-vortex-tube limit in which the viscous diffusion of vorticity is in balance with its axial stretching. This balance determines the order of the tube radius $(\nu / \sigma)^{1 / 2}$. Then the same argument as that for the diffusing vortex tube leads us to the estimate of the order of the total dissipation for the Burgers vortex tube $\Gamma^{2} \sigma$. Consequently, the total dissipation for the spirals could also overtake that for the Burgers vortex tube at the critical time

$$
S t_{c} \sim\left(\frac{\Gamma}{\nu}\right)^{2 / 3} \frac{\sigma}{S} .
$$

\section{Dissipation for spirals in turbulence}

In the present subsection, we discuss the contribution to turbulent energy dissipation from the spiral structures around the tubular vortices based upon the recent results from Jiménez et al.' $\mathrm{s}^{1,14}$ and Kida and Makihara's ${ }^{31}$ direct numerical simulations of forced isotropic turbulence and upon the present analytical results in Sec. III B.

First, suppose that the Reynolds number $\Gamma / \nu$ and the total axial length $l / L$ of tubular structures in turbulence scale, respectively, as

$$
\frac{\Gamma}{\nu} \sim R_{\lambda}^{a}, \quad \frac{l}{L} \sim R_{\lambda}^{b}
$$

for constant exponents $a$ and $b$, where $l$ is computed in an $L^{3}$ periodic box and $L\left(\sim R_{\lambda} \lambda\right)$ denotes the integral scale. The Taylor-microscale Reynolds number is defined as $R_{\lambda}$ $=u_{\mathrm{rms}} \lambda / \nu$, where $u_{\mathrm{rms}}$ is the rms velocity of turbulence, and $\lambda\left(\sim u_{\mathrm{rms}} / \omega_{\mathrm{rms}}\right)$ is the Taylor microscale. Recently Jiménez, Wray, Saffman, and Rogallo ${ }^{1}$ found that the cross-sectional radius of strong vortex tubes is of the order of the Kolmogorov microscale $\left(\nu / \omega_{\mathrm{rms}}\right)^{1 / 2}$, and its axial vorticity is $O\left(\omega_{\mathrm{rms}} R_{\lambda}^{1 / 2}\right)$, implying that the vortex Reynolds number scales with $R_{\lambda}^{1 / 2}$, i.e., $a=1 / 2$. Jiménez and Wray ${ }^{14}$ estimated the total axial length of the strong tubes as $l / L \sim R_{\lambda}$, i.e., $b$ $=1$. More recently Kida and Makihara ${ }^{31}$ examined the statistics of low-pressure vortices, and they found that $a \approx 0$ and $b \approx 2$ (strictly $a=0.15$ and $b=2.15$ ). The vortex Reynolds numbers of their low-pressure vortices are rather large $(\Gamma / \nu=107-124)$, and are nearly independent of $R_{\lambda}$. Their vortices are typical in the sense that not only the crosssectional radius but also the axial vorticity of the tubes scale 
with the Kolmogorov length and time. The differences in the values of $a$ and $b$ between Refs. 1 and 14 and Ref. 31 arise from the distinct identification criteria of tubular structures.

In turbulence, the axial strain rate $\sigma$ acting on tube-like structures is comparable to the background (or rms) vorticity $\omega_{\text {rms }}$ (see Ref. 1), which has been represented by the shear rate $S$ in this paper. Therefore, we suppose that $\sigma \sim S$ $\sim \omega_{\text {rms }}$, implying that the Burgers radius of tubular structures, $(\nu / \sigma)^{1 / 2}$, is of comparable order to the Kolmogorov microscale $\left(\nu / \omega_{\mathrm{rms}}\right)^{1 / 2}$. From (32) we have the critical time

$$
\omega_{\mathrm{rms}} t_{c} \sim\left(\frac{\Gamma}{\nu}\right)^{2 / 3} \sim R_{\lambda}^{2 a / 3} .
$$

The lifetimes of the tublar structures are of the order of the large-eddy-turnover time $L / u_{\mathrm{rms}}{ }^{14}$ Normalizing the critical time (34) with $L / u_{\text {rms }}$, we obtain

$$
\frac{u_{\mathrm{rms}} t_{c}}{L} \sim\left(\frac{\Gamma}{\nu}\right)^{2 / 3} R_{\lambda}^{-1} \sim R_{\lambda}^{2 a / 3-1} .
$$

It follows from (35) that $u_{\mathrm{rms}} t_{c} / L \sim R_{\lambda}^{-2 / 3}$ for the strong tubular vortices $(a=1 / 2)$ and that $u_{\mathrm{rms}} t_{c} / L \sim R_{\lambda}^{-1}$ for the typical vortices $(a=0)$. At either case the critical time would be much smaller than the large-eddy-turnover time at $R_{\lambda} \gg 1$.

Let us now discuss the volume fractions of the tubular and the spiral-layer structures, and their contribution rates to the turbulent energy dissipation. In the case of the tube-like structures, their cross section and total length are $O\left(\nu / \omega_{\text {rms }}\right)$ and $l$, respectively, and thus their volume fraction is estimated, from the latter scaling in (33), as

$$
\frac{\left(\nu / \omega_{\mathrm{rms}}\right) l}{L^{3}} \sim R_{\lambda}^{b-3} .
$$

Since the total-energy dissipation for the Burgers vortex tube per unit axial length is of the order $\Gamma^{2} \sigma$ as discussed in Sec. III B, we have the contribution rate to turbulent energy dissipation from the tubes,

$$
\frac{\left(\Gamma^{2} \omega_{\mathrm{rms}}\right) l}{\left(\nu \omega_{\mathrm{rms}}^{2} L^{3}\right.} \sim R_{\lambda}^{2 a+b-3},
$$

where we have used both the scalings in (33). In the case of the spiral layers, on the other hand, we recall that the volume per unit axial length of the spirals with highest dissipation is $O\left[(\Gamma / \nu)^{2 / 3} \nu t\right]$ as shown in Sec. III B. The lifetimes of the spiral structures are comparable with those of the tubular vortices, and thus we take the time average of the volume per unit axial length over the turnover time $L / u_{\mathrm{rms}}$. Because the spiral layers appear along the tube through the wrapping of vorticity lines around the tube, the total axial length of the spirals should be of the same order as that of the tube. Accordingly, we have the volume fraction of the spiral structures,

$$
\frac{\left[(\Gamma / \nu)^{2 / 3} \nu L / u_{\mathrm{rms}}\right] l}{L^{3}} \sim R_{\lambda}^{2 a / 3+b-2} .
$$

The contribution rate to the turbulent energy dissipation from the spirals is obtained, from (31) as

$$
\frac{\left[\nu^{2} \omega_{\mathrm{rms}}^{2}(\Gamma / \nu)^{4 / 3} \nu L / u_{\mathrm{rms}}\right] l}{\left(\nu \omega_{\mathrm{rms}}^{2}\right) L^{3}} \sim R_{\lambda}^{4 a / 3+b-2} .
$$

Equations (37) and (39) tell us that the contribution of the spirals to the turbulent energy dissipation dominates that of the tubes for $\Gamma / \nu \sim R_{\lambda}^{a}$ with $a<3 / 2$. Hence, the energy dissipation for the spiral structures would be much greater than that not only for the typical tubes $(a=0)$ but also for the strong tubes $(a=1 / 2)$.

It might be possible for the special case of $2 a+b-3=0$ that the contribution (37) of the vortex tubes to the turbulent dissipation would remain finite in the large-Reynoldsnumber limit $R_{\lambda} \rightarrow \infty$. In reality, however, the contribution (37) for both the typical $[(a, b)=(0,2)]$ and the strong $[(a, b)=(1 / 2,1)]$ tubes is of the order $R_{\lambda}^{-1}$, and therefore it disappears at $R_{\lambda} \gg 1$. $^{14}$

The finite contribution of the spirals (39) at $R_{\lambda} \gg 1$ requires the condition that $4 a / 3+b-2=0$. For the spirals around the strong tubes $[(a, b)=(1 / 2,1)]$ this condition is not satisfied $(4 a / 3+b-2=-1 / 3)$, and the contribution rate from the spirals would decay proportionally to $R_{\lambda}^{-1 / 3}$. For the spirals around the typical tubes $[(a, b)=(0,2)]$, on the other hand, it is strikingly interesting that the condition for the finite contribution is satisfied, which suggests that the spirals around the typical tubes play a crucial role in the turbulent dissipation at $R_{\lambda} \rightarrow \infty$. Because in the case of the typical tubes for $(a, b)=(0,2)$, the volume fraction (38) for the spirals would also be finite at $R_{\lambda} \gg 1$, one might think that the spiral structures around the typical tubes are indistinguishable from weak background turbulence. In this case, however, the vortex Reynolds number $\Gamma / \nu$ is finite but rather large (say, $\Gamma / \nu \sim 100$ ) and so the magnitude of the vorticity $\left[\omega_{\mathrm{rms}}(\Gamma / \nu)^{1 / 3}\right]$ and the local dissipation $\left[\nu \omega_{\mathrm{rms}}^{2}(\Gamma / \nu)^{2 / 3}\right]$ in the spirals are also large compared with those in the background field.

\section{EFFECTS OF SMALL TILT OF TUBE ON DISSIPATION}

In this section we consider the energy dissipation in the spiral vortex layers around a slightly tilted vortex tube at early-time evolution $S t|\alpha| \ll 1$ in order to demonstrate the effects of cross-axial shear on the spirals and their total dissipation. Moffatt, Kida, and Ohkitani ${ }^{16}$ have shown analytically the spatial distribution of the viscous dissipation rate around an intense vortex tube subject to a nonaxisymmetric irrotational strain. Although the (axisymmetric) circular ring region of high dissipation around the vortex tube is cross axially deformed by the effect of the nonaxisymmetric strain [see Figs. 1(d) and 1(f)], the deformation does not affect the total dissipation for the tube itself. ${ }^{16}$ In contrast with the dissipation for the tube, the cross-axial deformation of the spiral regions of high dissipation is expected to affect the total dissipation, because the interaction of a nonaxisymmetric strain with (nonaxisymmetric) spirals can yield an axisymmetric dissipative structure. 


\section{A. Asymptotic analysis at $S t|\alpha| \ll 1$}

If we consider asymptotic solutions to the vorticity equation (8) and to the Navier-Stokes equation (9) in the limit of a small tilt of a vortex tube $|\alpha| \ll 1$, the leading-order streamfunction and axial velocity coincide with (14) and (17) for $\alpha=0$ in Sec. III, respectively. Therefore, the asymptotic solutions to (8) and (9) may be written as

$$
\begin{aligned}
& \psi(r, \theta, t)=\Gamma\left[\psi_{0}(\eta)+\operatorname{St\alpha } \psi_{1}(\eta, \theta)+\cdots\right], \\
& u(r, \theta, t)=\operatorname{Sr}\left[u_{0}(\eta, \theta)+\operatorname{St\alpha } u_{1}(\eta, \theta)+\cdots\right]
\end{aligned}
$$

at early time $S t|\alpha| \ll 1$, where the leading-order streamfunction and axial velocity are represented, respectively, by $\Gamma \psi_{0}$ and $S r u_{0}$. Note that $\psi_{0}$ is defined by dividing (14) by the circulation $\Gamma$ and that $u_{0}=\operatorname{Re}\left[f(\eta) e^{-i \theta}\right]$.

By substituting the Taylor expansion, $\sin \alpha=\alpha-\frac{1}{6} \alpha^{3}$ $+\cdots$, and (40) with (14) to (8) and then putting the higherorder streamfunction as

$$
\psi_{1}=\operatorname{Re}\left[g(\eta) e^{-2 i \theta}\right],
$$

at the order $S t|\alpha|$ we have

$$
\begin{aligned}
g^{i v} & +2\left(\eta+\frac{1}{\eta}\right) g^{\prime \prime \prime}+\left(2-\frac{9}{\eta^{2}}\right) g^{\prime \prime}-\frac{1}{\eta}\left(10-\frac{9}{\eta^{2}}\right) g^{\prime}+\frac{16}{\eta^{2}} g \\
& +\frac{i}{\pi} \frac{\Gamma}{\nu}\left[\frac{1-e^{-\eta^{2}}}{\eta^{2}}\left(g^{\prime \prime}+\frac{1}{\eta^{\prime}} g^{\prime}-\frac{4}{\eta^{2}} g\right)+4 e^{-\eta^{2}} g\right] \\
& =\frac{4 i}{\pi} \eta^{2} e^{-\eta^{2}} .
\end{aligned}
$$

The inhomogeneous term in the right-hand side represents the cross-axial advection of the axial vorticity by the uniform shear flow. The boundary conditions to be imposed are that $\operatorname{tg}(\eta)$ is regular at $\eta=0$ and $\operatorname{tg}(\eta) \rightarrow 0$ as $\eta \rightarrow \infty$ [see (6) and (7)]. In Ref. 19 the solution to (43) has already been obtained at large Reynolds numbers $\Gamma / \nu \gg 1$ and it has been shown to be consistent with Moffatt, Kida, and Ohkitani's ${ }^{16}$ asymptotic solution for the nonaxisymmetric Burgers vortex tube if $\eta$ is replaced by $\frac{1}{2} r$. The streamfunction $g$ for the nonaxisymmetric Burgers tube represents a quadruple-type distribution that implies the deformation of the cross section of the tube into an elliptical shape.

By substituting (41) with (17) to (9) and putting the higher-order axial velocity as

$$
u_{1}=\operatorname{Re}\left\{i\left[f_{1}(\eta) e^{-i \theta}+f_{3}(\eta) e^{-3 i \theta}\right]\right\},
$$

at the order $S t|\alpha|$ we obtain, for the $\theta$-dependent part,

$$
f_{1}^{\prime \prime}+\left(2 \eta+\frac{3}{\eta}\right) f_{1}^{\prime}-4 f_{1}+i \frac{\Gamma}{2 \pi \nu} \frac{1-e^{-\eta^{2}}}{\eta^{2}} f_{1}=\zeta(\eta)
$$

and obtain, for the $3 \theta$-dependent part,

$$
\begin{aligned}
f_{3}^{\prime \prime} & +\left(2 \eta+\frac{3}{\eta}\right) f_{3}^{\prime}-4\left(1+\frac{2}{\eta^{2}}\right) f_{3}+3 i \frac{\Gamma}{2 \pi \nu} \frac{1-e^{-\eta^{2}}}{\eta^{2}} f_{3} \\
& =\chi(\eta) .
\end{aligned}
$$

The inhomogeneous terms in the right-hand side, $\zeta$ and $\chi$, are given, respectively, by

$$
\begin{aligned}
& \zeta=-\frac{\Gamma}{\nu} \frac{1}{\eta^{2}}\left(\bar{f} g+\eta \overline{f^{\prime}} g+\frac{1}{2} \eta \bar{f} g^{\prime}\right)+\eta \overline{f^{\prime}}-4 i \operatorname{Im}(f), \\
& \chi=-\frac{\Gamma}{\nu} \frac{1}{\eta^{2}}\left(f g+\eta f^{\prime} g-\frac{1}{2} \eta f g^{\prime}\right)+\eta f^{\prime},
\end{aligned}
$$

where hereafter the overline is used to denote the complex conjugate. The first terms in the right-hand side of (47) and (48) originate from the cross-axial advection of the axial velocity by the flow induced by the above quadruple (i.e., $g$ ), while the second (and third) terms come from the cross-axial advection by the uniform shear flow. The boundary conditions to be imposed are that $r t f_{1}(\eta)$ and $r t f_{3}(\eta)$ are regular at $\eta=0$, and $r t f_{1}(\eta) \rightarrow 0$ and $r t f_{3}(\eta) \rightarrow 0$ as $\eta \rightarrow \infty$ [see (6) and (7)].

In the following we seek the asymptotic solutions to (45) and (46) at $\Gamma / \nu \gg 1$. Let us first examine the order of the inhomogeneous terms (47) and (48). The solution $f$ to (18) (and so $f^{\prime}$ ) decreases to zero exponentially as $\Gamma / \nu \rightarrow \infty$ up to the region $\eta \sim(\Gamma / \nu)^{1 / 4}$ (see Ref. 19), whereas $f$ is given by (19) in the far region $\eta \gg(\Gamma / \nu)^{1 / 4}$ at large $\Gamma / \nu$. Therefore, $f$ and $f^{\prime}$ are estimated to be $O(1)$ and $O\left(\eta^{-3} \Gamma / \nu\right)$, respectively. On the other hand, the large-Reynolds-number asymptotic solution $g$ to (43) is of the order $(\Gamma / \nu)^{-1}$ in the near region $\eta \sim 1$ and is of the order $\eta^{-2}(\Gamma / \nu)^{-1}$ at $\eta \gg 1$ (see Ref. 16). It follows from the orders of $f$ and $g$ that the inhomogeneous terms (47) and (48) are exponentially small as $\Gamma / \nu \rightarrow \infty$ up to the region $\eta \sim(\Gamma / \nu)^{1 / 4}$, while at $\eta \gg(\Gamma / \nu)^{1 / 4}, \zeta$ and $\chi$ can be written as

$$
\begin{aligned}
& \zeta \approx \eta \bar{f}^{\prime}-4 i \operatorname{Im}(f) \approx i \frac{R_{\Gamma}}{2 \eta^{2}} \exp \left(-i \frac{R_{\Gamma}}{4 \eta^{2}}-\frac{R_{\Gamma}^{2}}{48 \eta^{6}}\right) \\
&-4 i \sin \left(\frac{R_{\Gamma}}{4 \eta^{2}}\right) \exp \left(-\frac{R_{\Gamma}^{2}}{48 \eta^{6}}\right), \\
& \chi \approx \eta f^{\prime} \approx-i \frac{R_{\Gamma}}{2 \eta^{2}} \exp \left(i \frac{R_{\Gamma}}{4 \eta^{2}}-\frac{R_{\Gamma}^{2}}{48 \eta^{6}}\right),
\end{aligned}
$$

where the solution (19) has been used. We note that the contribution of the advection by the quadruple has disappeared from (49) and (50).

Accordingly, if we consider the far region $\eta \gg(\Gamma / \nu)^{1 / 4}$ and thus all the terms that include $e^{-\eta^{2}}$ can be neglected, we may rewrite (45) and (46) as

$$
\begin{aligned}
& f_{1}^{\prime \prime}+\left(2 \eta+\frac{3}{\eta}\right) f_{1}^{\prime}-\left(4-i \frac{\Gamma}{2 \pi \nu} \frac{1}{\eta^{2}}\right) f_{1}=\zeta, \\
& f_{3}^{\prime \prime}+\left(2 \eta+\frac{3}{\eta}\right) f_{3}^{\prime}-\left[4+\left(8-3 i \frac{\Gamma}{2 \pi \nu}\right) \frac{1}{\eta^{2}}\right] f_{3}=\chi,
\end{aligned}
$$

where the inhomogeneous terms $\zeta$ and $\chi$ are given by (49) and (50). At large Reynolds numbers $R_{\Gamma}=\Gamma /(2 \pi \nu) \gg 1$ the Wentzel-Kramers-Brillouin (WKB) method can be applied to (51) and (52), and consequently we obtain the full expressions of the asymptotic solutions, 


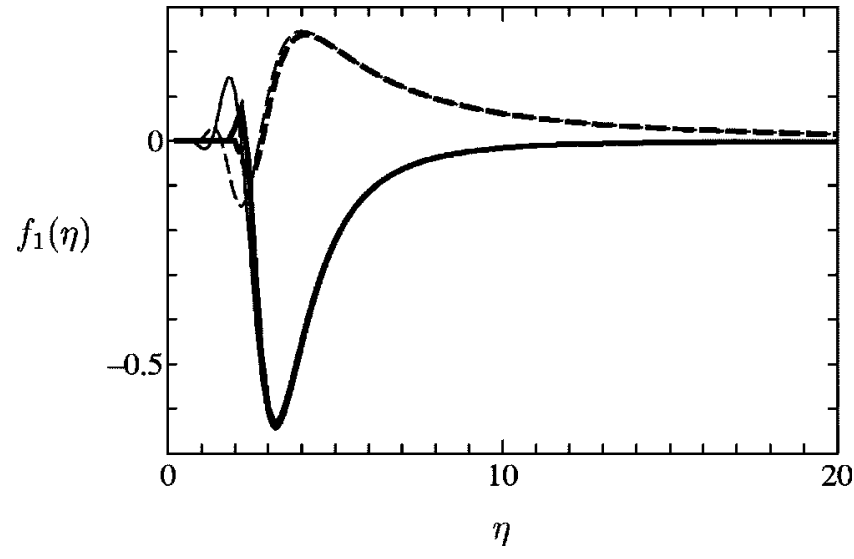

FIG. 4. Solution $f_{1}$ against similarity variable $\eta$ at vortex Reynolds number $\Gamma /(2 \pi \nu)=100$. The solid curves represent the real part of $f_{1}$, while the dashed curves represent the imaginary part. The thick and the thin curves denote the asymptotic solutions (53) and (54), and the numerical ones to the full equation (45), respectively.

$$
\begin{aligned}
& \operatorname{Re}\left(f_{1}\right)=\frac{3}{4}\left[\cos \left(\frac{R_{\Gamma}}{4 \eta^{2}}\right)-\frac{4 \eta^{2}}{R_{\Gamma}} \sin \left(\frac{R_{\Gamma}}{4 \eta^{2}}\right)\right] \exp \left(-\frac{R_{\Gamma}^{2}}{48 \eta^{6}}\right), \\
& \operatorname{Im}\left(f_{1}\right)=\frac{1}{4} \sin \left(\frac{R_{\Gamma}}{4 \eta^{2}}\right) \exp \left(-\frac{R_{\Gamma}^{2}}{48 \eta^{6}}\right)
\end{aligned}
$$

and

$$
\begin{aligned}
f_{3}= & -\left(1-\frac{i \eta^{2}}{2 R_{\Gamma}}\right) \exp \left(i \frac{R_{\Gamma}}{4 \eta^{2}}-\frac{R_{\Gamma}^{2}}{48 \eta^{6}}\right) \\
& -\frac{i \eta^{2}}{2 R_{\Gamma}} \exp \left(i \frac{3 R_{\Gamma}}{4 \eta^{2}}-\frac{3 R_{\Gamma}^{2}}{16 \eta^{6}}\right) .
\end{aligned}
$$

The solutions (53)-(55) represent the effects of the crossaxial uniform shear on the spiral vortex layers wrapped around a vortex filament. Figures 4 and 5, respectively, compare the asymptotic solutions, (53) and (54) for the $\theta$-dependent part and (55) for the $3 \theta$-dependent part, with the

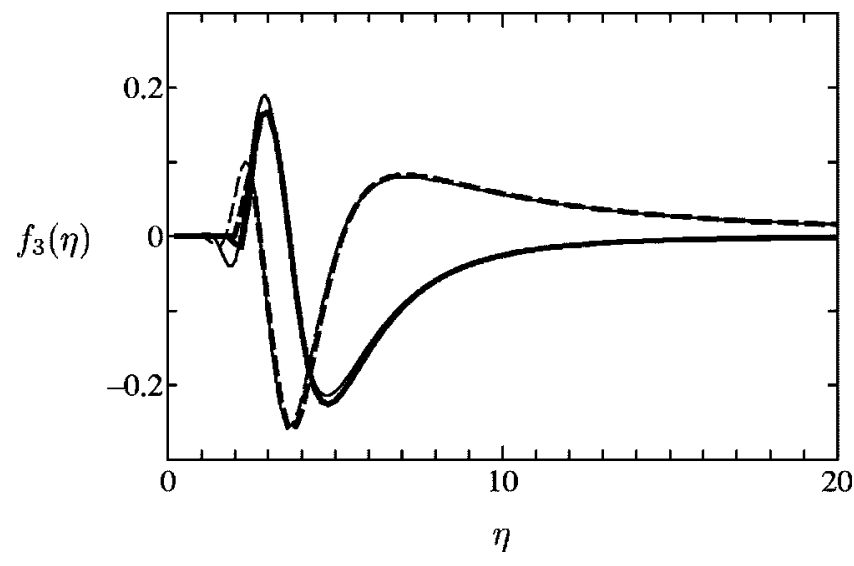

FIG. 5. Solution $f_{3}$ against similarity variable $\eta$ at vortex Reynolds number $\Gamma /(2 \pi \nu)=100$. The solid curves represent the real part of $f_{3}$, while the dashed curves represent the imaginary. The thick and the thin curves denote the asymptotic solutions (55) and the numerical ones to the full equation (46), respectively. numerical ones to the full equations (45) and (46) at moderate Reynolds number $\Gamma /(2 \pi \nu)=100$. The solid and the dashed curves represent the real and the imaginary part of the solutions. The thick and the thin curves denote the asymptotic and the numerical solutions. It can be seen in Figs. 4 and 5 that even at moderate Reynolds number $\Gamma /(2 \pi \nu)=100$, the asymptotic solutions are in good agreement with the numerical ones except for relatively small values of $\eta$. The region of disagreement should shrink as $\Gamma /(2 \pi \nu)$ is increased.

\section{B. Cross-axially sheared spirals}

We now discuss the effects of the cross-axial shear on the viscous dissipation around the slightly tilted vortex tube for $|\alpha| \ll 1$. By substituting the expansion (40) up to the order $S t|\alpha|$ with (14) and (42) into the dissipation rate (12), in which $\sin \alpha$ is expanded as $\sin \alpha=\alpha-\frac{1}{6} \alpha^{3}+\cdots$, we obtain the dissipation for the tilted tube,

$$
\begin{aligned}
D_{T}= & \nu\left(\frac{\Gamma}{4 \pi \nu t}\right)^{2}\left[\frac{1}{\eta^{2}}-\left(1+\frac{1}{\eta^{2}}\right) e^{-\eta^{2}}\right]^{2} \\
& +\nu \frac{S t \alpha}{8 \pi}\left(\frac{\Gamma}{\nu t}\right)^{2}\left[\frac{1}{\eta^{2}}-\left(1+\frac{1}{\eta^{2}}\right) e^{-\eta^{2}}\right] \\
& \times \operatorname{Re}\left[\left(g^{\prime \prime}-\frac{1}{\eta^{\prime}}+\frac{4}{\eta^{2}} g\right) e^{-2 i \theta}\right] \\
& -\nu \frac{S \alpha}{2 \pi} \frac{\Gamma}{\nu t}\left[\frac{1}{\eta^{2}}-\left(1+\frac{1}{\eta^{2}}\right) e^{-\eta^{2}}\right] \cos 2 \theta .
\end{aligned}
$$

For the dissipation caused by the spiral vortex layers around the tilted tube, on the other hand, we substitute the expansion (41) up to the order $S t|\alpha|$ with (17) and (44) into the dissipation rate (13), in which $\cos ^{2} \alpha$ is expanded as $\cos ^{2} \alpha=1$ $-\alpha^{2}+\cdots$, to obtain

$$
\begin{aligned}
D_{S}= & \nu S^{2}\left[\frac{1}{2}\left|\eta f^{\prime}\right|^{2}+\frac{1}{2} \eta\left(|f|^{2}\right)^{\prime}+|f|^{2}-1\right] \\
& +\nu S^{2} \operatorname{Re}\left[\eta f^{\prime}\left(f+\frac{1}{2} \eta f^{\prime}\right) e^{-2 i \theta}\right] \\
& -2 \nu S^{3} t \alpha \operatorname{Re}\left\{i\left[\frac{1}{2} \eta^{2} f^{\prime} \bar{f}_{1}^{\prime}+\frac{1}{2} \eta\left(f \bar{f}_{1}\right)^{\prime}+f \bar{f}_{1}\right]\right\} \\
& +2 \nu S^{3} t \alpha \operatorname{Re}\left\{i \left[\frac{1}{2} \eta^{2}\left(\bar{f}^{\prime} f_{3}^{\prime}+f^{\prime} f_{1}^{\prime}\right)+\frac{1}{2} \eta\left(\bar{f} f_{3}+f f_{1}\right)^{\prime}\right.\right. \\
& \left.\left.+2 \bar{f} f_{3}\right] e^{-2 i \theta}\right\}+2 \nu S^{3} t \alpha \operatorname{Re}\left\{i \left[\frac{1}{2} \eta^{2} f^{\prime} f_{3}^{\prime}+\frac{1}{2} \eta\left(f f_{3}\right)^{\prime}\right.\right. \\
& \left.\left.-f f_{3}\right] e^{-4 i \theta}\right\} .
\end{aligned}
$$

The first terms in the right-hand side of (56) and (57) represent the leading-order dissipation (see Sec. III A), whereas the others denote the higher-order correction.

In Figs. 1(c), 1(d), 1(e), and 1(f) are shown the spatial distributions, in the cross-axial plane, of the magnitude of the cross-axial vorticity $\left[(\partial u / \partial r)^{2}+r^{-2}(\partial u / \partial \theta)^{2}\right]^{1 / 2}$ and of the viscous dissipation $D_{T}+D_{S}$, respectively, for the cyclonic 
case $\alpha=+0.2$ and for the anticyclonic case $\alpha=-0.2$. The cross-axial vorticity [(c) and (e)] and the viscous dissipation $[(\mathrm{d})$ and (f) $]$ have been obtained at time $S t=2.94(S t|\alpha|$ $=0.59$ ) by numerically solving the full equations (43), (45), and (46). The tube and the spirals undergo the cross-axial shear $S y \sin \alpha \boldsymbol{e}_{z}$ [see (1)], and in the cyclone [(c) and (d)] for $\alpha>0$ \{or the anticyclone [(e) and (f)] for $\alpha<0\}$ they are sheared from the left to the right (or from the right to the left) in the upper half $(y>0)$ of the panels while from the right to the left (or from the left to the right) in the lower half ( $y$ $<0$ ) of the panels.

Let us first discuss the effect of the cross-axial shear on the circular ring of high dissipation, which is caused by the vortex tube [see Fig. 1(b)]. As demonstrated analytically in Ref. 16, if an intense tube $(\Gamma / \nu \gg 1)$ is subject to a nonaxisymmetric cross-axial strain, a vortex core is deformed into an elliptical shape, the major axis of which turns at an angle $\pi / 4$ from the principal direction of the greater rate of strain. The deformation of the vortex core brings about two maxima of a viscous dissipation rate on the major axis of the ellipse. We can confirm the resulting two maxima in Figs. 1(d) and 1(f), though the turning angles of the two maxima are less than $\pi / 4$ which is the asymptotic value at $\Gamma / \nu \rightarrow \infty$. We should note that the spatial structure of the energy dissipation $D_{T}$ for the vortex tube is not different between the cyclone $(\alpha>0)$ and the anticyclone $(\alpha<0)$ except for difference in the argument, in the $(y, z)$ plane, of the structures.

In contrast with the spatial structure of high dissipation caused by the vortex tube, the double spirals of high (vorticity and) dissipation are cross axially deformed into different shapes depending on the sign of $\alpha$, as shown in Figs. 1(c), 1(d), 1(e), and 1(f). If $\alpha>0$ (the cyclonic case), the radial spacing of the isocontours of the axial velocity in the outermost strong spirals is widened by the cross-axial shear to reduce the radial gradient of the axial velocity, so that the azimuthal vorticity and the corresponding viscous dissipation rate are reduced [cf. Figs. 1(a), 1(b), 1(c), and 1(d)]. If $\alpha$ $<0$ (the anticyclonic case), on the other hand, the spacing of the isocontours of the axial velocity is tightened, which leads to a remarkable enhancement of the azimuthal vorticity and the dissipation rate [cf. Figs. 1(a) and 1(b), and Figs. 1(e) and $1(\mathrm{f})]$.

\section{Reduction and enhancement of total dissipation}

As shown in Sec. IV B, the viscous dissipation for the spirals is locally reduced or enhanced depending on the direction of the cross-axial shear (i.e., the sign of $\alpha$ ). Here we analytically evaluate the effect of the cross-axial shear on the total dissipation for the spirals. The total dissipation for the spirals at $|\alpha| \ll 1$ is expressed, using (57), as

$$
\int_{0}^{\infty} \int_{0}^{2 \pi} D_{S} r \mathrm{~d} r \mathrm{~d} \theta=8 \pi \nu^{2} S^{2} I_{0} t-16 \pi \nu^{2} S^{3} \alpha I_{1} t^{2}
$$

where the first term in the right-hand side represents the leading order (see Sec. III B), and the second represents the higher-order correction which comes from the axisymmetric contribution in (57). The integral $I_{1}$ is defined by

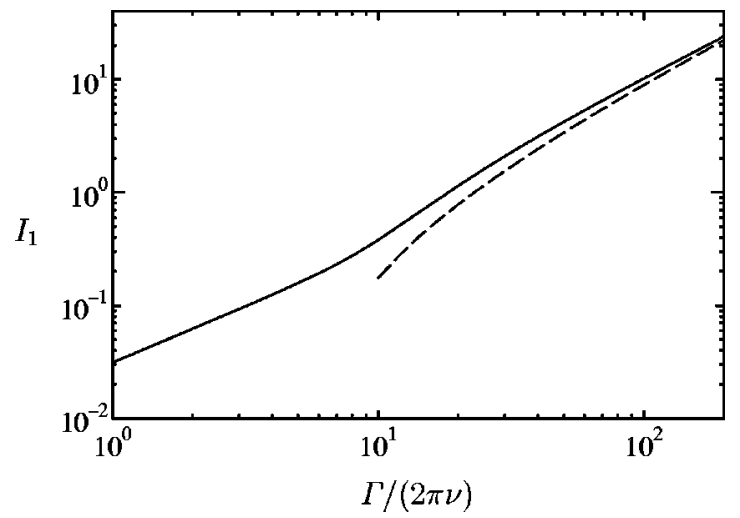

FIG. 6. Integral $I_{1}$ in (59) against vortex Reynolds number $\Gamma /(2 \pi \nu)$. The solid curve represents the numerical computation of (59) for the numerical solutions, $f$ and $f_{1}$, to (18) and (45). The dashed line denotes the largeReynolds-number asymptotics (65).

$$
I_{1}=\int_{0}^{\infty} \operatorname{Re}\left\{i\left[\frac{1}{2} \eta^{2} f^{\prime} \bar{f}_{1}^{\prime}+\frac{1}{2} \eta\left(f \bar{f}_{1}\right)^{\prime}+f \bar{f}_{1}\right]\right\} \eta d \eta .
$$

For large Reynolds numbers $R_{\Gamma}=\Gamma /(2 \pi \nu) \gg 1, f$ and $f_{1}$ are, respectively, given by (19), (53), and (54), so that the integral (59) may be rewritten as

$$
I_{1} \approx \frac{R_{\Gamma}}{32} I_{2}+\frac{R_{\Gamma}}{16}
$$

where

$$
I_{2}=3 \int_{0}^{\infty}\left[-\frac{2}{\xi^{2}} \sin \xi+\frac{1}{\xi}(1+\cos \xi)\right] \exp \left(-\frac{\xi^{3}}{3 R_{\Gamma}}\right) d \xi .
$$

If we differentiate (61) with respect to $R_{\Gamma}$, we have

$$
\frac{d I_{2}}{d R_{\Gamma}}=\frac{1}{R_{\Gamma}}+\frac{1}{R_{\Gamma}^{2}} \int_{0}^{\infty}\left(-2 \xi \sin \xi+\xi^{2} \cos \xi\right) \exp \left(-\frac{\xi^{3}}{3 R_{\Gamma}}\right) d \xi .
$$

Therefore, at $R_{\Gamma} \gg 1$ we obtain

$$
\frac{d I_{2}}{d R_{\Gamma}} \approx \frac{1}{R_{\Gamma}},
$$

and the integral (61) turns out to be expressed as

$$
I_{2} \approx \ln R_{\Gamma}+C,
$$

where $C$ is an integral constant to be determined numerically. Substitution of (64) into (60) yields

$$
I_{1} \approx \frac{R_{\Gamma}}{32} \ln R_{\Gamma}+\frac{C+2}{32} R_{\Gamma},
$$

where the first term represents the primary contribution, and the coefficient, $(C+2) / 32$, of the second correction term is evaluated to be $-0.0546(C=-3.75)$ by the numerical integration of (61).

Figure 6 compares the large-Reynolds-number asymptotic form (65) with the numerical computation of (59) for the numerical solutions, $f$ and $f_{1}$, to the full equations (18) and (45). In the numerical computation of (45), the nu- 
merical solution $g$ to (43) is substituted into its inhomogeneous term. It can be seen in the figure that the integral $I_{1}$ takes a positive value, and that at large $\Gamma / \nu, I_{1}$ is well described by the asymptotic form (65). Accordingly, it turns out analytically that the effects of the cross-axial shear [the second term in the right-hand side of (58)] reduce (or enhance) the total-energy dissipation for the spirals in the cyclonic case $\alpha>0$ (or the anticyclonic case $\alpha<0$ ). At $\Gamma / \nu \gg 1$ the primary contribution from the cross-axial shear to the total dissipation (58) can be written explicitly as

$$
-16 \pi \nu^{2} S^{3} \alpha I_{1} t^{2} \approx-\frac{1}{4} \alpha \nu S^{3} \Gamma \ln \left(\frac{\Gamma}{2 \pi \nu}\right) t^{2},
$$

and its comparison with the leading-order total dissipation, i.e., the first term in the right-hand side of (58), implies that the contribution of the cross-axial shear increases slower than the leading-order contribution, which is proportional to $(\Gamma / 2 \pi \nu)^{4 / 3}$ [see (28)], with increasing $\Gamma /(2 \pi \nu)$. The leadingorder contribution (28) dominates the higher-order contribution (66) at $S t|\alpha| \ll(\Gamma / \nu)^{1 / 3} / \ln (\Gamma / \nu)$.

\section{CONCLUDING REMARKS}

In this paper we have investigated analytically the energy dissipation in double spiral vortex layers which are formed around a straight vortex tube through the wrap and stretch of vorticity lines of background uniform shear flow with a shear rate $S$. The vortex tube is inclined in the direction of the uniform shear vorticity at a small angle $\alpha$ from the direction of the uniform shear velocity. The tube, which starts with a vortex filament of circulation $\Gamma$ at initial time $t=0$, is diffused under the action of viscosity. The spiral vortex layers are dominated by the azimuthal vorticity, and thus they are different from the Lundgren spirals ${ }^{17}$ in which the vorticity aligns with a vortex tube. Full analytical expressions of not only the solutions for the spiral vortex layers but also their contribution to the total-energy dissipation per unit axial length have been obtained at large Reynolds numbers $\Gamma / \nu \gg 1$ and at early-time evolution $S t|\alpha| \ll 1$. These expressions are independent of the inner structure of the vortex tube, and they are expected to be useful for understanding spiral structures in turbulent flows.

The total-energy dissipation for the spiral vortex layers around the longitudinal vortex tube of $\alpha=0$ is evaluated asymptotically to be $1.29 \pi \nu^{2} S^{2}(\Gamma / 2 \pi \nu)^{4 / 3} t$ at $\Gamma / \nu \gg 1$, whereas that for the diffusing tube is expressed as $\Gamma^{2} /(8 \pi t)$. The total-energy dissipation for the spiral layers exceeds that for the diffusing tube after the critical time $S t$ $=0.623(\Gamma / 2 \pi \nu)^{1 / 3}$. The total dissipation for the Burgers vortex tube subject to the axial strain $\sigma$ is of the order $\Gamma^{2} \sigma$, and therefore the total dissipation for the spirals could also overtake that for the Burgers tube at the critical time $S t$ $\sim(\Gamma / \nu)^{2 / 3} \sigma / S$.

We have related the present analytical results with the energy dissipation for tubular and spiral structures in turbulence. We suppose that the vortex Reynolds number and the total axial length of tubular (and spiral) structures in turbulence are scaled with the Taylor-miscroscale Reynolds number $R_{\lambda}$ as $\Gamma / \nu \sim R_{\lambda}^{a}$ and $l / L \sim R_{\lambda}^{b}$, respectively. The axial strain $\sigma$ acting on tubular vortices is known to be comparable with the background vorticity $\omega_{\text {rms }}$ which is represented by $S$ in this analysis (i.e., $\sigma \sim S \sim \omega_{\text {rms }}$ ). Accordingly the total dissipation for the spiral structures would be greater than that for the tubular structure (i.e., the Burgers tube) after the critical time, normalized by the large-eddy-turnover time $L / u_{\text {rms }}$, of the order $R_{\lambda}^{2 a / 3-1}$. The contribution rate of the tubes to turbulent energy dissipation would be of the order $R_{\lambda}^{2 a+b-3}$, while by taking time-average over the turnover time $L / u_{\mathrm{rms}}$ the contribution rate of the spirals has been estimated to be of the order $R_{\lambda}^{4 a / 3+b-2}$. For $a<3 / 2$ it has been suggested at $R_{\lambda} \gg 1$ that the critical time is much smaller than the turnover time, and that the contribution rate from the spirals to the turbulent energy dissipation dominates that from the tubes. The direct numerical simulations of forced isotropic turbulence have provided us with the scaling exponents, $(a, b)$ $=(1 / 2,1)$ for strong tubular vortices ${ }^{1,14}$ and $(a, b)=(0,2)$ for typical (low-pressure) vortices. ${ }^{31}$ In the case of either vortex, a significant contributor to the turbulent energy dissipation should be the spiral layers rather than the tubes. Recently the high-dissipation structures were found to have dimensions $1.7 \pm 0.1$, suggesting structures in the form of layers or ribbons. ${ }^{37}$ We have also suggested that even in the largeReynolds-number limit $R_{\lambda} \rightarrow \infty$ the contribution rate from the spirals to the turbulent dissipation remains finite under the condition of $4 a / 3+b-2=0$. This condition is satisfied in the case of the typical vortices $[(a, b)=(0,2)] .{ }^{31}$

If the vortex tube is tilted $(\alpha \neq 0)$, the tube and the spirals undergo the cross-axial shear. The spirals of high dissipation are cross axially deformed into different shapes depending on the sign of $\alpha$, so that the energy dissipation in the spirals is locally reduced (or enhanced) in the cyclonic case $\alpha>0$ (or the anticyclonic case $\alpha<0$ ). The local reduction and enhancement of the disspiation rate have been shown analytically to affect the total dissipation for the spirals. The primary effect of the cross-axial shear on the total dissipation for the spirals has been evaluated asymptotically to be $-\frac{1}{4} \alpha \nu S^{3} \Gamma \ln (\Gamma / 2 \pi \nu) t^{2}$ at $S t|\alpha| \ll 1$ for $\Gamma / \nu \gg 1$.

In uniformly sheared turbulence ${ }^{2}$ and near-wall turbulence, ${ }^{4}$ quasistreamwise vortices are often observed to slightly tilt in the spanwise direction. In isotropic turbulence tubular vortices (and spiral vortex layers around them) may undergo the cross-axial shear (or nonaxisymmetric strain). ${ }^{12,16}$ The cross-axial shear would play different roles in the turbulent dissipation depending on configuration. For instance, the spiral vortex layers around the quasistreamwise vortex of the cyclonic inclination are considered to be less dissipative than those of the anticyclonic inclination, which might be related to dominance of the cyclonic structures observed in shear flow turbulence.

It is interesting and important whether the spiral structures of high azimuthal vorticity are stable or not. The linear stability analyses of an elliptic jet ${ }^{38}$ and a corrugated vortex sheet ${ }^{39}$ showed that the curvature of vorticity lines on a sheet suppresses the Kelvin-Helmholtz instability. Not only the curvature of the spiral layers but also the mutual constraint between the wrapped layers are expected to reduce the instability. However, the problem of their stability is left for a future study. 


\section{ACKNOWLEDGMENTS}

The author would like to express his thanks to Professor M. Nagata for his encouragement. He appreciates helpful discussions with Professor S. Kida, Professor S. Yanase, and Professor M. Tanaka in the development of this study. He also gives credit for the numerical assistance to his former graduate students, H. Kiyama and H. Yagi. This work was partially supported by a Grant-in-Aid for Scientific Research from Japan Society for the Promotion of Science and by the Center of Excellence for Research and Education on Complex Functional Mechanical Systems (COE program of the Ministry of Education, Culture, Sport, Science and Technology of Japan).

${ }^{1}$ J. Jiménez, A. A. Wray, P. G. Saffman, and R. S. Rogallo, "The structure of intense vorticity in homogeneous isotropic turbulence," J. Fluid Mech. 255, 65 (1993).

${ }^{2}$ S. Kida and M. Tanaka, "Dynamics of vortical structures in a homogeneous shear flow," J. Fluid Mech. 274, 43 (1994).

${ }^{3}$ M. Tanahashi, T. Miyauchi, and K. Matsuoka, "Coherent fine scale structure in temporally developing turbulent mixing layers," in Proceedings of 2nd International Symposium on Turbulence, Heat and Mass Transfer, edited by K. Hanjalic and T. W. Peeters (Delft University Press, Delft, 1997), p. 461.

${ }^{4}$ J. Jeong, F. Hussain, W. Schoppa, and J. Kim, "Coherent structures near the wall in a turbulent channel flow," J. Fluid Mech. 332, 185 (1997).

${ }^{5}$ E. D. Siggia, "Numerical study of small scale intermittency in threedimensional turbulence," J. Fluid Mech. 107, 375 (1981).

${ }^{6}$ R. M. Kerr, "Higher-order derivative correlation and the alignment of small scale structures in isotropic turbulence," J. Fluid Mech. 153, 31 (1985).

${ }^{7}$ I. Hosokawa and K. Yamamoto, "Fine structure of a directly simulated isotropic turbulence," J. Phys. Soc. Jpn. 58, 20 (1989).

${ }^{8}$ Z.-S. She, E. Jackson, and S. A. Orszag, "Intermittent vortex structures in homogeneous isotropic turbulence," Nature (London) 344, 226 (1991).

${ }^{9}$ G. R. Ruetsch and M. R. Maxey, "Small scale features of vorticity and passive scalar fields in homogeneous isotropic turbulence," Phys. Fluids A 3, 1587 (1991).

${ }^{10} \mathrm{~A}$. Vincent and M. Meneguzzi, "The spatial structure and statistical properties of homogeneous turbulence," J. Fluid Mech. 225, 1 (1991).

${ }^{11}$ D. Douady, Y. Couder, and M. E. Brachet, "Direct observation of the intermittency of intense vorticity filaments in turbulence," Phys. Rev. Lett. 67, 983 (1991).

${ }^{12} \mathrm{~S}$. Kida and K. Ohkitani, "Spatio-temporal intermittency and instability of a forced turbulence," Phys. Fluids A 4, 1018 (1992).

${ }^{13}$ S. Kida, "Tube-like structures in turbulence," Lecture Notes in Numerical Applied Analysis 12, 137 (1993).

${ }^{14}$ J. Jiménez and A. A. Wray, "On the characteristics of vortex filaments in isotropic turbulence," J. Fluid Mech. 373, 255 (1998).

${ }^{15} \mathrm{H}$. Tennekes, "Simple model for the small-scale structure of turbulence," Phys. Fluids 11, 669 (1968).

${ }^{16}$ H. K. Moffatt, S. Kida, and K. Ohkitani, "Stretched vortices-the sinews of turbulence; large-Reynolds-number asymptotics," J. Fluid Mech. 259, 241 (1994).

${ }^{17}$ T. S. Lundgren, "Strained spiral vortex model for turbulent fine structure," Phys. Fluids 25, 2193 (1982).

${ }^{18} \mathrm{D}$. W. Moore, "The interaction of a diffusing line vortex and aligned shear flow," Proc. R. Soc. London, Ser. A 399, 367 (1985).

${ }^{19}$ G. Kawahara, S. Kida, M. Tanaka, and S. Yanase, "Wrap, tilt and stretch of vorticity lines around a strong thin straight vortex tube in a simple shear flow," J. Fluid Mech. 353, 115 (1997).

${ }^{20}$ S. Corrsin, "Turbulent dissipation fluctuations," Phys. Fluids 5, 1301 (1962).

${ }^{21}$ G. R. Reutsh and M. R. Maxey, "The evolution of small-scale structures in homogeneous turbulence," Phys. Fluids A 4, 2747 (1992).

${ }^{22}$ A. Vincent and M. Meneguzzi, "On the dynamics of vorticity tubes in homogeneous turbulence," J. Fluid Mech. 258, 245 (1994).

${ }^{23}$ T. Passot, H. Politano, P.-L. Sulem, J. R. Angilella, and M. Meneguzzi, "Instability of strained vortex layers and vortex tube formation in homogeneous turbulence," J. Fluid Mech. 282, 313 (1995).

${ }^{24}$ T. S. Lundgren, "A small-scale turbulence model," Phys. Fluids A 5, 1472 (1993).

${ }^{25}$ A. D. Gilbert, "A cascade interpretation of Lundgren's stretched spiral vortex model for turbulent fine structure," Phys. Fluids A 5, 2831 (1993).

${ }^{26}$ D. I. Pullin and T. S. Lundgren, "Axial motion and scalar transport in stretched spiral vortices," Phys. Fluids 13, 2553 (2001).

${ }^{27}$ K. Ohkitani, "A survey on a class of exact solutions of the Navier-Stokes equations and a model for turbulence," Publ. Res. Inst. Math. Sci. 40, 1267 (2004).

${ }^{28}$ S. Kida and H. Miura, "Double spirals around a tubular vortex in turbulence,” J. Phys. Soc. Jpn. 69, 3466 (2000).

${ }^{29} \mathrm{~K}$. Horiuti, "A classification method for vortex sheet and tube structures in turbulent flows," Phys. Fluids 13, 3756 (2001).

${ }^{30} \mathrm{~K}$. Horiuti, in Advances in Turbulence X, edited by H. I. Andersson and P.-Å. Krogstad (CIMNE, Barcelona, 2004), p. 35.

${ }^{31} \mathrm{~S}$. Kida and T. Makihara, "Reynolds-number dependence of low-pressure vortex in turbulence," in Proceedings of International Symposium on Dynamics and Statistics of Coherent Structures in Turbulence: Roles of Elementary Vortices, edited by S. Kida (2002), p. 229.

${ }^{32}$ G. Kawahara, S. Kida, S. Yanase, and M. Tanaka, in Advances in Turbulence VIII, edited by C. Dopazo (CIMNE, Barcelona, 2000), p. 733.

${ }^{33}$ G. Kawahara, H. Kiyama, and H. Yagi, "Vorticity stretching and energy dissipation around a straight vortex tube in a uniform shear flow," JSME Int. J., Ser. B 44, 369 (2001).

${ }^{34}$ R. Verzicco, J. Jiménez, and P. Orlandi, "On steady columnar vortices under local compression," J. Fluid Mech. 299, 367 (1995).

${ }^{35} \mathrm{H}$. Miura and S. Kida, "Identification of tubular vortices in turbulence," J. Phys. Soc. Jpn. 66, 1331 (1997).

${ }^{36}$ P. E. Dimotakis, "The mixing transition in turbulent flows," J. Fluid Mech. 409, 69 (2000).

${ }^{37}$ F. Moisy and J. Jiménez, "Geometry and clustering of intense structures in isotropic turbulence," J. Fluid Mech. 513, 111 (2004).

${ }^{38}$ D. G. Crighton, "Instability of an elliptic jet," J. Fluid Mech. 59, 665 (1973).

${ }^{39}$ G. Kawahara, J. Jiménez, M. Uhlmann, and A. Pinelli, "Linear instability of a corrugated vortex sheet-a model for streak instability," J. Fluid Mech. 483, 315 (2003). 